\title{
Global robust adaptive output regulation for nonlinear systems of relative degree up to two
}

\author{
Zhiyong Chen And Jie Huang
}

\begin{abstract}
In this paper, we study the global robust output regulation problem for an uncertain nonlinear lower triangular system subject to an uncertain exosystem. Under some standard assumptions, the problem can be transformed into a robust adaptive stabilization problem of a well defined system called augmented system. In order to deal with the unknown parameter of the exosystem, we construct an internal model consisting of two dynamic compensators. As a result, the augmented system contains both the static and dynamic uncertainties and the dynamic uncertainty is not inputto-state stable. Then, we develop a recursive approach to construct the controller.
\end{abstract}

\section{Introduction}

Consider a lower-triangular system of relative degree two described as follows,

$$
\begin{aligned}
\dot{q}_{o} & =\kappa_{o}\left(q_{o}, q_{1}, v, w\right) \\
\dot{q}_{1} & =\kappa_{1}\left(q_{o}, q_{1}, v, w\right)+q_{2} \\
\dot{q}_{2} & =\kappa_{2}\left(q_{o}, q_{1}, q_{2}, v, w\right)+u \\
e & =q_{1}-q_{d}(v, w),
\end{aligned}
$$

where $q_{o} \in \mathbb{R}^{n_{o}}$ and $q_{i} \in \mathbb{R}$ are the states, $u \in \mathbb{R}$ is the input, and $e \in \mathbb{R}$ is the output representing the tracking error. The disturbance and/or reference signal $v \in \mathbb{R}^{q}$ is produced by an exosystem described by

$$
\dot{v}=A_{1}(\sigma) v, \quad v(0)=v_{o}
$$

The unknown parameters $w \in \mathbb{R}^{p_{1}}$ and $\sigma \in \mathbb{R}^{p_{2}}$ are assumed to be in some known compact sets. It is also assumed that all functions in the system (1.1) 
are polynomial in $\left(q_{o}, q_{1}, q_{2}, v\right)$. We will consider the problem described as follows.

Global Robust Adaptive Output Regulation Problem: For given $\mathbb{V}, \mathbb{W}$, and $\mathbb{S}$, which are compact subsets of $\mathbb{R}^{q}, \mathbb{R}^{p_{1}}$, and $\mathbb{R}^{p_{2}}$ containing the origins, respectively, find a state feedback controller such that, for all $v(t) \in \mathbb{V}, w \in$ $\mathbb{W}$, and $\sigma \in \mathbb{S}$, the trajectories of the closed-loop system, starting from any initial states, exist and are bounded for all $t>0$, and satisfy $\lim _{t \rightarrow \infty} e(t)=0$.

Various versions of the problem have been extensively studied for the case where the exosystem is exactly known in, for example, 1 15. When the exosystem contains uncertainty, the signal $v$ represents the combination of constant and sinusoids with unknown amplitudes, phases and frequencies. This more practical problem was first studied for a simple nonlinear system in [6], and was subsequently further pursued for more complex systems in [7-12], etc. It is worth mentioning that the semi-global output regulation problem for a class of lower triangular systems was studied in [12] via output feedback control. Here, we consider the same system as the system studied in [12. However, our objective is global output regulation by state feedback. Even though we only consider the case where the relative degree is two, the iterative nature of our approach allows it to be extended to a higher relative degree case with more complicated notation and assumptions.

Typically, the output regulation problem is approached in two steps. In the first step, an appropriate dynamic compensator called internal model is found. Attaching the internal model to the given plant leads to an augmented system, and the internal model is such that the stabilization solution of the augmented system leads to the output regulation solution of the original plant. As a result, the second step will be centered on stabilizing the augmented system. When the exosystem is exactly known, the stabilization problem of the augmented system can often be handled by various robust control techniques. However, when the exosystem contains uncertain parameters, these uncertain parameters will enter the augmented systems and they cannot be handled by robust control techniques. Therefore, for this case, adaptive control techniques have to be further introduced to handle this type of the unknown parameters.

In all existing papers dealing with the output regulation problem of nonlinear systems with an unknown exosystem, a common feature is that only one dynamic compensator corresponding to the input needs to be constructed. As a result, the only unknown parameter vector that needs to be estimated by the adaptive control technique appears in one single equation containing the input. In contrast, in the present case, we need to construct $r$ dynamic compensators corresponding to $r-1$ measurable states and one 
input where $r$ is the relative degree. Thus, the resulting augmented system will also be much more complex in that $r$ unknown parameter vectors appear in $r$ equations and all these unknown parameter vectors need to be estimated by the adaptive control technique. Such type of systems has never been encountered before. In this paper, we will propose a new robust adaptive design approach to overcome this difficulty. This approach needs to recursively construct the control law $r$ times, and each recursion necessitates a dynamic coordinate transformation which leads to a newly augmented system with more complex parameter uncertainty. This phenomenon is called "propagation of uncertainties". To capture the essential mechanism of our design method, and avoid tedious notation and assumptions, we only consider the lower triangular system (1.1) whose relative degree is two.

The rest of the paper is organized as follows. Section 2 provides a typical construction of an adaptive internal model to deal with uncertain exosystems. Based on the adaptive internal model and a new robust adaptive control approach, the global robust output regulation problem formulated in this paper is solved in Section 3. Finally, the paper is concluded in Section 4 .

\section{Problem conversion}

To begin with, let us recall from the general framework established in [5] that the solvability of the robust output regulation problem relies on an appropriate internal model. To ascertain the existence of the desired internal model, we will list the following four assumptions.

Assumption 2.1. For all $\sigma \in \mathbb{S}$, the exosystem is assumed to be neutrally stable in the sense that all the eigenvalues of $A_{1}(\sigma)$ are simple and have zero real parts.

Assumption 2.2. There exists $\mathbf{q}_{o}(v, w, \sigma)$, a polynomial function in $v$ with coefficients depending on $w$ and $\sigma$ such that,

$$
\frac{\partial \mathbf{q}_{o}(v, w, \sigma)}{\partial v} A_{1}(\sigma) v=\kappa_{o}\left(\mathbf{q}_{o}(v, w, \sigma), q_{d}(v, w), v, w\right)
$$

for all $v \in \mathbb{V}, w \in \mathbb{W}$, and $\sigma \in \mathbb{S}$.

Assumption 2.3. There exists a sufficiently smooth function $V\left(q_{o}\right)$ bounded by some class $\mathcal{K}_{\infty}$ polynomial functions, such that, along the trajectories of 
$\dot{q}_{o}=\kappa_{o}\left(q_{o}, q_{1}, v, w\right)$,

$$
\frac{d V\left(q_{o}\right)}{d t} \leq-\left\|q_{o}\right\|^{2}+\pi\left(q_{1}\right)
$$

for some polynomial positive definite function $\pi$.

Assumption 2.4. There exist polynomial functions $\varkappa_{2}$ and $\varkappa_{3}$ in $\left(q_{o}, q_{1}\right.$, $\left.q_{2}, v\right)$ such that

$$
\kappa_{2}\left(q_{o}, q_{1}, q_{2}, v, w\right)=\varkappa_{2}\left(q_{o}, q_{1}, q_{2}, w\right)+\varkappa_{3}\left(q_{o}, q_{1}, v, w\right) .
$$

Remark 2.1. Assumption 2.1 means that the solution of the exosystem is a sum of finitely many sinusoidal functions. Typically, $\sigma$ represents the frequencies of these sinusoidal functions. Under Assumption 2.2, we can define, for all $v \in \mathbb{V}, w \in \mathbb{W}$, and $\sigma \in \mathbb{S}$,

$$
\begin{aligned}
\mathbf{q}_{1}(v, w) & =q_{d}(v, w) \\
\mathbf{q}_{2}(v, w, \sigma) & =\frac{\partial \mathbf{q}_{1}(v, w)}{\partial v} A_{1}(\sigma) v-\kappa_{1}\left(\mathbf{q}_{o}(v, w, \sigma), \mathbf{q}_{1}(v, w), v, w\right) \\
\mathbf{u}(v, w, \sigma) & =\frac{\partial \mathbf{q}_{2}(v, w, \sigma)}{\partial v} A_{1}(\sigma) v-\kappa_{2}\left(\mathbf{q}_{o}(v, w, \sigma), \mathbf{q}_{1}(v, w), \mathbf{q}_{2}(v, w, \sigma), v, w\right)
\end{aligned}
$$

which constitute the solution of the regulator equations for the system composed of 1.1 and $(1.2)$ for all $v \in \mathbb{V}, w \in \mathbb{W}$, and $\sigma \in \mathbb{S}$. And the solutions are polynomial in $v$ with coefficients depending on $w$ and $\sigma$. Assumption 2.3 implies that the system (1.1) is minimum phase. Assumption 2.4 excludes cross terms between $q_{2}$ and $v$ in $\kappa_{2}\left(q_{o}, q_{1}, q_{2}, v, w\right)$ so that the uncertainty term $l_{2}\left(y_{2}, \mu\right)$ in the augmented system (2.8) satisfies the linear parameterization condition which is needed for the employment of the adaptive control technique later.

Since $\mathbf{q}_{2}(v, w, \sigma)$ and $\mathbf{u}(v, w, \sigma)$ are polynomial in $v$ with coefficients depending on $w$ and $\sigma$, there exist integers $r_{1}$ and $r_{2}$, and matrices $\Phi_{i}(\sigma) \in$ $\mathbb{R}^{r_{i} \times r_{i}}$ such that

$$
\dot{\vartheta}_{i}=\Phi_{i}(\sigma) \vartheta_{i}, \quad \xi_{i}=\left[\begin{array}{llll}
1 & 0 & \cdots & 0
\end{array}\right] \vartheta_{i}, \quad i=1,2
$$

where $\vartheta_{i}(v, w, \sigma):=\operatorname{col}\left(\xi_{i}, \dot{\xi}_{i}, \ldots, \xi_{i}^{\left(r_{i}-1\right)}\right), \quad i=1,2, \quad \xi_{1}=\mathbf{q}_{2}(v, w, \sigma), \quad \xi_{2}=$ $\mathbf{u}(v, w, \sigma)$. Moreover, all the eigenvalues $\Phi_{i}(\sigma)$ are simple with zero real parts. 
Next, pick any controllable pairs $\left(M_{i}, N_{i}\right)$ with $M_{i} \in \mathbb{R}^{r_{i} \times r_{i}}, N_{i} \in \mathbb{R}^{r_{i} \times 1}$, and $M_{i}$ Hurwitz, and solve $T_{i}(\sigma)$ from the Sylvester equation $T_{i}(\sigma) \Phi_{i}(\sigma)-$ $M_{i} T_{i}(\sigma)=N_{i}\left[\begin{array}{llll}1 & 0 & \cdots & 0\end{array}\right]$. Let

$$
\begin{aligned}
\theta_{i}(v, w, \sigma) & :=T_{i}(\sigma) \vartheta_{i}(v, w, \sigma) \\
E_{i}(\sigma) & :=T_{i}(\sigma) \Phi_{i}(\sigma) T_{i}^{-1}(\sigma) \\
\Psi_{i}(\sigma) & :=\left[\begin{array}{llll}
1 & 0 & \cdots & 0
\end{array}\right] T_{i}^{-1}(\sigma)
\end{aligned}
$$

and

$$
\begin{aligned}
\theta(v, w, \sigma) & =\operatorname{col}\left(\theta_{1}(v, w, \sigma), \theta_{2}(v, w, \sigma)\right), \\
\alpha(\sigma, \theta) & =\operatorname{block} \operatorname{diag}\left(E_{1}(\sigma) \theta_{1}, E_{2}(\sigma) \theta_{2}\right), \\
\beta(\sigma, \theta) & =\operatorname{col}\left(\Psi_{1}(\sigma) \theta_{1}, \Psi_{2}(\sigma) \theta_{2}\right), \\
\mathrm{X} & =\operatorname{block} \operatorname{diag}\left(\mathrm{X}_{1}, \mathrm{X}_{2}\right), \\
\mathrm{X} & =M, N, T .
\end{aligned}
$$

Then, it can be verified that the triplet $\{\theta(v, w, \sigma), \alpha(\sigma, \theta), \beta(\sigma, \theta)\}$ satisfies

$$
\dot{\theta}(v, w, \sigma)=\alpha(\sigma, \theta), \quad \xi=\beta(\sigma, \theta), \quad \xi:=\operatorname{col}\left(\xi_{1}, \xi_{2}\right)
$$

The triplet $\{\theta(v, w, \sigma), \alpha(\sigma, \theta), \beta(\sigma, \theta)\}$ is called a steady-state generator of the system (1.1) and 1.2 with the output $\operatorname{col}\left(q_{2}, u\right)$. Furthermore, let

$$
\dot{\eta}=M \eta+N \operatorname{col}\left(q_{2}, u\right), \quad \eta:=\operatorname{col}\left(\eta_{1}, \eta_{2}\right)
$$

which defines the internal model for system $(1.1)$ and $(1.2)$ with the output $\operatorname{col}\left(q_{2}, u\right)$. The composition of the system (1.1) and the internal model 2.3 is called an augmented system.

If $\sigma$ were known, then based on the framework in [5], we could further perform on the augmented system (1.1) and (2.3) the following input and state transformations

$$
\begin{gathered}
\bar{u}=u-\Psi_{2}(\sigma) \eta_{2}, \quad z_{i}=\eta_{i}-\theta_{i}-N_{i} x_{i}, \quad i=1,2, \\
x_{o}=q_{o}-\mathbf{q}_{o}(v, w, \sigma), \quad x_{1}=e, \quad x_{2}=q_{2}-\Psi_{1}(\sigma) \eta_{1}
\end{gathered}
$$

to obtain a transformed augmented system in the following form:

$$
\begin{aligned}
& \dot{x}_{o}=f_{o}\left(x_{o}, x_{1}, d\right), \quad \dot{z}_{1}=M_{1} z_{1}+\gamma_{1}\left(\chi_{1}, d\right) \\
& \dot{x}_{1}=f_{1}\left(\zeta_{1}, \chi_{1}, d\right)+x_{2}, \quad \dot{z}_{2}=M_{2} z_{2}+\gamma_{2}\left(\zeta_{1}, \chi_{2}, d\right) \\
& \dot{x}_{2}=f_{2}\left(\zeta_{2}, \chi_{2}, d\right)+\bar{u}
\end{aligned}
$$


where $\chi_{1}:=\operatorname{col}\left(x_{o}, x_{1}\right), \chi_{2}:=\operatorname{col}\left(x_{o}, x_{1}, x_{2}\right), \zeta_{1}:=z_{1} \zeta_{2}:=\operatorname{col}\left(z_{1}, z_{2}\right), d=$ $\operatorname{col}(v, w, \sigma)$, and $\mu=\operatorname{col}(w, \sigma)$. Also, the functions $f_{i}$ and $\gamma_{i}$ satisfy $f_{i}(0,0, d)$ $=0$ for $i=0,1,2$, and $\left.\gamma_{(} 0, d\right)=0$ and $\gamma_{2}(0,0, d)=0$. The system (2.5) is in a standard lower triangular form with static uncertainty $d$ and dynamic uncertainty $\left(x_{o}, z_{1}, z_{2}\right)$. It was shown in [5] that it is possible to globally stabilize the system (2.5) by a state feedback controller $\bar{u}=g_{u}\left(x_{1}, x_{2}\right)$ based on the small gain approach. Moreover, this controller will lead to the following controller

$$
u=\Psi_{2}(\sigma) \eta_{2}+g_{u}\left(e, q_{2}-\Psi_{1}(\sigma) \eta_{1}\right), \quad \dot{\eta}=M \eta+N \operatorname{col}\left(x_{2}, u\right)
$$

that solves the global output regulation problem of the original system.

Unfortunately, when $\sigma$ is unknown, the control law 2.6 is not implementable. To overcome the difficulty caused by the uncertain exosystem, we will instead consider performing on $(1.1)$ and $(2.3)$ the following coordinate transformation:

$$
\begin{aligned}
z_{i} & =\eta_{i}-\theta_{i}-N_{i} x_{i}, \quad i=1,2, \\
x_{o} & =q_{o}-\mathbf{q}_{o}(v, w, \sigma), \quad x_{1}=e, \quad x_{2}=q_{2} .
\end{aligned}
$$

This transformation leads to the following transformed augmented system:

$$
\begin{aligned}
\dot{x}_{o} & =f_{o}\left(x_{o}, x_{1}, d\right) \\
\dot{z}_{1} & =M_{1} z_{1}+\gamma_{1}\left(\chi_{1}, d\right) \\
\dot{x}_{1} & =f_{1}\left(\zeta_{1}, \chi_{1}, d\right)+l_{1}\left(y_{1}, \mu\right)+x_{2}, \\
\dot{z}_{2} & =M_{2} z_{2}+\gamma_{2}\left(\zeta_{1}, \chi_{2}, d\right)+h_{2}\left(\bar{y}_{2}, \mu\right) \\
\dot{x}_{2} & =f_{2}\left(\zeta_{2}, \chi_{2}, d\right)+l_{2}\left(y_{2}, \mu\right)+u
\end{aligned}
$$

where $\bar{y}_{1}=\operatorname{col}\left(e, q_{o}, q_{1}\right), y_{1}=\operatorname{col}\left(e, q_{o}, q_{1}, \eta_{1}\right), \bar{y}_{2}=\operatorname{col}\left(e, q_{o}, q_{1}, q_{2}, \eta_{1}\right)$, and $y_{2}=\operatorname{col}\left(e, q_{o}, q_{1}, q_{2}, \eta_{1}, \eta_{2}\right)$. Various functions in 2.8 are described as follows:

$$
\begin{aligned}
f_{o}\left(x_{o}, x_{1}, d\right) & =\kappa_{o}\left(q_{o}, q_{1}, v, w\right)-\kappa_{o}\left(\mathbf{q}_{o}, \mathbf{q}_{1}, v, w\right) \\
\gamma_{1}\left(\chi_{1}, d\right) & =M_{1} N_{1} x_{1}-N_{1} A_{1} \\
f_{1}\left(\zeta_{1}, \chi_{1}, d\right) & =A_{1}+\Psi_{1}(\sigma) \eta_{1}-\Psi_{1}(\sigma) \theta_{1} \\
\gamma_{2}\left(\zeta_{1}, \chi_{2}, d\right) & =M_{2} N_{2} x_{2}-N_{2} A_{2}+N_{2} \Psi_{1}(\sigma) E_{1}(\sigma)\left(N_{1} x_{1}+z_{1}\right) \\
f_{2}\left(\zeta_{2}, \chi_{2}, d\right) & =A_{2}-\Psi_{1}(\sigma) E_{1}(\sigma)\left(N_{1} x_{1}+z_{1}\right)+\Psi_{2}(\sigma)\left(N_{2} x_{2}+z_{2}\right)
\end{aligned}
$$


and

$$
\begin{aligned}
l_{1}\left(y_{1}, \mu\right) & =-\Psi_{1}(\sigma) \eta_{1} \\
h_{2}\left(\bar{y}_{2}, \mu\right) & =-N_{2} B-N_{2} \Psi_{1}(\sigma) E_{1}(\sigma) \eta_{1} \\
l_{2}\left(y_{2}, \mu\right) & =B+\Psi_{1}(\sigma) E_{1}(\sigma) \eta_{1}-\Psi_{2}(\sigma) \eta_{2}
\end{aligned}
$$

where

$$
\begin{aligned}
A_{1}:= & \kappa_{1}\left(q_{o}, q_{1}, v, w\right)-\kappa_{1}\left(\mathbf{q}_{o}, \mathbf{q}_{1}, v, w\right) \\
A_{2}:= & \varkappa_{2}\left(q_{o}, q_{1}, \Psi_{1}(\sigma) \eta_{1}, w\right)-\varkappa_{2}\left(\mathbf{q}_{o}, \mathbf{q}_{1}, \mathbf{q}_{2}, w\right) \\
& +\varkappa_{3}\left(q_{o}, q_{1}, v, w\right)-\varkappa_{3}\left(\mathbf{q}_{o}, \mathbf{q}_{1}, v, w\right) \\
B= & \varkappa_{2}\left(q_{o}, q_{1}, q_{2}, w\right)-\varkappa_{2}\left(q_{o}, q_{1}, \Psi_{1}(\sigma) \eta_{1}, w\right) .
\end{aligned}
$$

Remark 2.2. Like the case where $\sigma$ is known, if the system 2.8 can be globally stabilized in the sense that the states of the closed-loop are globally bounded and $\lim _{t \rightarrow \infty} x_{1}(t)=0$, then the resulting controller will lead to a controller that solves the global output regulation problem for the original system (1.1) with $\sigma$ unknown. Therefore, for the rest of this paper, we only need to focus on the stabilization of $(2.8)$.

Remark 2.3. The system (2.8) appears much more complicated than the system 2.5) in that the left hand side of 2.8) contains such terms as $l_{1}, l_{2}$ and $h_{2}$. Owing to the presence of these terms, $\left(\zeta_{2}, \chi_{2}\right)=0$ is not the equilibrium point of the system any more. For this reason, we refer to these terms as equilibrium point perturbing (e.p.p.) terms in what follows. The presence of the e.p.p. terms greatly complicates the global stabilization of 2.8). In particular, the dynamic uncertainty $z_{2}$ does not satisfy a certain input-tostate stability condition. Therefore, the small gain approach as employed in [5] does not apply to 2.8. As a result, we need to develop a specific technique to handle the stabilization problem of 2.8 in the next section.

Remark 2.4. When using the output feedback control to deal with the output regulation problem of the system (1.1), the control law does not utilize the state variable $q_{2}$. Therefore, there is no need to construct an internal model corresponding to $q_{2}$. As a result, instead of using the transformation (2.7), one can use the following transformation

$$
\begin{aligned}
\bar{u} & =u-\Psi_{2}(\sigma) \eta_{2}, \quad z_{i}=\eta_{i}-\theta_{i}-N_{i} x_{i}, \quad i=1,2, \\
x_{o} & =q_{o}-\mathbf{q}_{o}(v, w, \sigma), \quad x_{1}=e, \quad x_{2}=q_{2}-\mathbf{q}_{2}(v, w, \sigma) .
\end{aligned}
$$


Under this transformation, the augmented system (1.1) and (2.3) will be transformed into a system similar to 2.8 but with the terms $l_{1}$ and $l_{2}$ vanishing. Such a system is still much simpler than 2.8). However, even this much simpler system cannot be globally stabilized by output feedback control. That is why only semi-global output regulation was considered in [12].

\section{Main result}

In this section, we will consider the stabilization problem of the system (2.8). First, as seen in [13], under Assumption 2.3, there exist polynomial functions $\alpha_{i}(\cdot), i=1,2$, such that, under the new coordinate

$$
\tilde{x}_{o}=x_{o}, \quad \tilde{x}_{1}=x_{1}, \quad \tilde{x}_{2}=x_{2}-\alpha_{1}\left(\tilde{x}_{1}\right), \quad \tilde{x}_{3}=x_{3}-\alpha_{2}\left(\tilde{x}_{2}\right),
$$

the system (2.8) with $l_{1}=0, l_{2}=0$ and $h_{2}=0$ becomes

$$
\begin{aligned}
\dot{\tilde{x}}_{0} & =f_{0}\left(\tilde{x}_{o}, \tilde{x}_{1}, d\right) \\
\dot{z}_{i} & =M_{i} z_{i}+\varphi_{i}\left(\zeta_{i-1}, \tilde{\chi}_{i}, d\right) \\
\dot{\tilde{x}}_{i} & =\phi_{i}\left(\zeta_{i}, \tilde{\chi}_{i}, d\right)+\alpha_{i}\left(\tilde{x}_{i}\right)+\tilde{x}_{i+1}, \quad i=1,2
\end{aligned}
$$

for some polynomial functions $\varphi_{i}$ and $\phi_{i}$. Moreover, there exist positive definite and radially unbounded functions $V\left(\zeta_{2}\right)$ and $W\left(\tilde{\chi}_{2}\right)=\sum_{i=0}^{2} W_{i}\left(\tilde{x}_{i}\right)$ with $\tilde{\chi}_{2}:=\operatorname{col}\left(\tilde{x}_{o}, \tilde{x}_{1}, \tilde{x}_{2}\right)$, such that, along the trajectories of the system (3.1),

$$
\frac{d\left(V\left(\zeta_{2}\right)+W\left(\tilde{\chi}_{2}\right)\right)}{d t} \leq-k\left(\zeta_{2}, \tilde{\chi}_{2}\right)
$$

for some positive definite function $k(\cdot, \cdot)$.

Next, we will turn to deal with the nontrivial terms $l_{1}, l_{2}$ and $h_{2}$. We first consider the upper subsystem governing $\operatorname{col}\left(x_{o}, z_{1}, x_{1}\right)$. To apply the adaptive scheme, we note the fact that

$$
\Psi_{1}(\sigma)=\omega_{1}^{\top}(\mu) \bar{\Psi}_{1}
$$

for a constant matrix $\bar{\Psi}_{1}$ and a column function vector $\omega_{1}(\mu)$. As a result, we have the following linear parameterization property of $l_{1}\left(y_{1}, \mu\right)$ :

$$
l_{1}\left(y_{1}, \mu\right)=-\Psi_{1}(\sigma) \eta_{1}=\rho_{1}\left(y_{1}\right) \omega_{1}(\mu)
$$

for a row vector function $\rho_{1}\left(y_{1}\right)=-\eta_{1}^{\top} \bar{\Psi}_{1}^{\top}$. Consider the following coordinate transformation:

$$
\bar{x}_{2}=x_{2}+\rho_{1}\left(y_{1}\right) \hat{\omega}_{1}-\alpha_{1}\left(x_{1}\right)
$$


with the function $\alpha_{1}(\cdot)$ given above, and the vector $\hat{\omega}_{1}$ used to estimate $\omega_{1}(\mu)$. In particular, $\hat{\omega}_{1}$ is generated by an update law

$$
\dot{\hat{\omega}}_{1}=\psi_{1}\left(y_{1}\right)=k_{1}\left(d W_{1}\left(x_{1}\right) / d x_{1}\right) \rho_{1}^{\top}\left(y_{1}\right), \quad k_{1}>0 .
$$

As a result, the $x_{1}$-subsystem is in the following form:

$$
\begin{aligned}
\dot{x}_{1} & =f_{1}\left(\zeta_{1}, \chi_{1}, d\right)+\alpha_{1}\left(x_{1}\right)-\rho_{1}\left(y_{1}\right) \tilde{\omega}_{1}+\bar{x}_{2} \\
& =\phi_{1}\left(\zeta_{1}, \chi_{1}, d\right)+\alpha_{1}\left(x_{1}\right)-\rho_{1}\left(y_{1}\right) \tilde{\omega}_{1}+\bar{x}_{2}
\end{aligned}
$$

with $\tilde{\omega}_{1}=\hat{\omega}_{1}-\omega_{1}$.

From above, we see that the notation

$$
\left(\bar{\chi}_{i}\right)-\left(\varrho_{i}, \varpi_{i}\right)-\left(\bar{\zeta}_{i}, s_{i}\right)-\left(\rho_{i}, \omega_{i}\right)-\left(\psi_{i}\right)-\left(\hat{\omega}_{i}\right)
$$

has been defined for $i=1$ with

$$
\begin{aligned}
& \bar{\chi}_{1}=\operatorname{col}\left(x_{o}, \bar{x}_{1}\right), \quad \bar{x}_{1}=x_{1}, \\
& \varrho_{1} \in \mathbb{R}^{o}, \quad \varpi_{1} \in \mathbb{R}^{o}, \quad \bar{\zeta}_{1}=\bar{z}_{1}=z_{1}, \quad s_{1} \in \mathbb{R}^{0} .
\end{aligned}
$$

Before moving to the full system (2.8), we need to define the notations 3.3 for $i=2$ as follows.

- Let $\bar{\chi}_{2}:=\operatorname{col}\left(x_{o}, \bar{x}_{1}, \bar{x}_{2}\right)$.

- Define

$$
\begin{aligned}
\hbar_{2}\left(\bar{y}_{2}, \hat{\omega}_{1}, \mu\right)= & \gamma_{2}\left(\zeta_{1}, \chi_{2}, d\right)-\gamma_{2}\left(\bar{\zeta}_{1}, x_{o}, \bar{x}_{1}, \bar{x}_{2}+\alpha_{1}\left(\bar{x}_{1}\right), d\right)+h_{2}\left(\bar{y}_{2}, \mu\right) \\
= & -M_{2} N_{2} \rho_{1}\left(y_{1}\right) \hat{\omega}_{1}+N_{2} \varkappa_{2}\left(q_{o}, q_{1}, q_{2}, w\right) \\
& -N_{2} \varkappa_{2}\left(q_{o}, q_{1}, \Psi_{1}(\sigma) \eta_{1}, w\right)-N_{2} \Psi_{1}(\sigma) E_{1}(\sigma) \eta_{1} .
\end{aligned}
$$

Since all functions are in polynomial form, the function $\hbar_{2}\left(\bar{y}_{2}, \hat{\omega}_{1}, \mu\right)$ satisfies the following linearly parameterization property:

$$
\hbar_{2}\left(\bar{y}_{2}, \hat{\omega}_{1}, \mu\right)=\varrho_{2}\left(\bar{y}_{2}, \hat{\omega}_{1}\right) \varpi_{2}(\mu)
$$

for a sufficiently smooth function matrix $\varrho_{2}$ and a column function vector $\varpi_{2}$.

- Let $s_{2}$ be a square matrix governed by $\dot{s}_{2}=M_{2} s_{2}+\varrho_{2}\left(\bar{y}_{2}, \hat{\omega}_{1}\right)$, and let $\bar{\zeta}_{2}:=\operatorname{col}\left(\bar{z}_{1}, \bar{z}_{2}\right)$ where $\bar{z}_{2}=z_{2}-s_{2} \varpi_{2}(\mu)$. 
- Define $\bar{\rho}_{1}\left(y_{2}, \mu\right)=-\dot{\eta}_{1}^{\top} \bar{\Psi}_{1}^{\top}=-\left(M_{1} \eta_{1}+N_{1} x_{2}\right)^{\top} \bar{\Psi}_{1}^{\top}$. Then, the derivative of $\rho_{1}\left(y_{1}\right)$ is $d \rho_{1}\left(y_{1}\right) / d t=\bar{\rho}_{1}\left(y_{2}, \mu\right)$. Let

$$
\begin{aligned}
& \ell_{2}\left(y_{2}, s_{2}, \hat{\omega}_{1}, \mu\right) \\
= & f_{2}\left(\zeta_{2}, \chi_{2}, d\right)-f_{2}\left(\bar{\zeta}_{2}, x_{o}, \bar{x}_{1}, \bar{x}_{2}+\alpha_{1}\left(\bar{x}_{1}\right), d\right)+l_{2}\left(y_{2}, \mu\right) \\
& +\left(\partial \alpha_{1}\left(\bar{x}_{1}\right) / \partial \bar{x}_{1}\right) \rho_{1}\left(y_{1}\right) \tilde{\omega}_{1}(\mu)+\bar{\rho}_{1}\left(y_{2}, \mu\right) \hat{\omega}_{1}(\mu)+\rho_{1}\left(y_{1}\right) \psi_{1}\left(y_{1}\right) \\
= & -\Psi_{2}(\sigma) N_{2} \rho_{1}\left(y_{1}\right) \hat{\omega}_{1}+\varkappa_{2}\left(q_{o}, q_{1}, q_{2}, w\right)-\varkappa_{2}\left(q_{o}, q_{1}, \Psi_{1}(\sigma) \eta_{1}, w\right) \\
& +\Psi_{2}(\sigma) s_{2} \varpi_{2}(\mu)+l_{2}\left(y_{2}, \mu\right)+\left(\partial \alpha_{1}\left(\bar{x}_{1}\right) / \partial \bar{x}_{1}\right) \rho_{1}\left(y_{1}\right) \tilde{\omega}_{1}(\mu) \\
& +\bar{\rho}_{1}\left(y_{2}, \mu\right) \hat{\omega}_{1}(\mu)+\rho_{1}\left(y_{1}\right) \psi_{1}\left(y_{1}\right) .
\end{aligned}
$$

Clearly, the function $\ell_{2}\left(y_{2}, s_{2}, \hat{\omega}_{1}, \mu\right)$ is linearly parameterized in the sense that

$$
\ell_{2}\left(y_{2}, s_{2}, \hat{\omega}_{1}, \mu\right)=\rho_{2}\left(y_{2}, s_{2}, \hat{\omega}_{1}\right) \omega_{2}(\mu)
$$

for a sufficiently smooth row vector function $\rho_{2}$ and a column function vector $\omega_{2}$.

- Let $\psi_{2}\left(y_{2}, s_{2}, \hat{\omega}_{1}\right)=k_{2}\left(d W_{2}\left(\bar{x}_{2}\right) / d \bar{x}_{2}\right) \rho_{2}^{\top}\left(y_{2}, s_{2}, \hat{\omega}_{1}\right), k_{2}>0$.

- Let $\hat{\omega}_{2}$ be a vector variable governed by $\dot{\hat{\omega}}_{2}=\psi_{2}\left(y_{2}, s_{2}, \hat{\omega}_{1}\right)$.

With the above notation, the $z_{2}$-subsystem can be rewritten as follows,

$$
\begin{aligned}
\dot{z}_{2} & =M_{2} z_{2}+\gamma_{2}\left(\bar{\zeta}_{1}, x_{o}, \bar{x}_{1}, \bar{x}_{2}+\alpha_{1}\left(\bar{x}_{1}\right), d\right)+\hbar_{2}\left(\bar{y}_{2}, \hat{\omega}_{1}, \mu\right) \\
& =M_{2} z_{2}+\varphi_{2}\left(\bar{\zeta}_{1}, \bar{\chi}_{2}, d\right)+\hbar_{2}\left(\bar{y}_{2}, \hat{\omega}_{1}, \mu\right) .
\end{aligned}
$$

We can define the following dynamic coordinate transformation

$$
\bar{z}_{2}=z_{2}-s_{2} \varpi_{2}(\mu), \quad \dot{s}_{2}=M_{2} s_{2}+\varrho_{2}\left(\bar{y}_{2}, \hat{\omega}_{1}\right)
$$

under which, we have

$$
\dot{\bar{z}}_{2}=M_{2} \bar{z}_{2}+\varphi_{2}\left(\bar{\zeta}_{1}, \bar{\chi}_{2}, d\right)
$$

Furthermore, with the controller $u=-\rho_{2}\left(y_{2}, s_{2}, \hat{\omega}_{1}\right) \hat{\omega}_{2}+\alpha_{2}\left(\bar{x}_{2}\right)$, we have

$$
\begin{aligned}
\dot{\bar{x}}_{2}= & f_{2}\left(\bar{\zeta}_{2}, x_{o}, \bar{x}_{1}, \bar{x}_{2}+\alpha_{1}\left(\bar{x}_{1}\right), d\right) \\
& -\left(\partial \alpha_{1}\left(\bar{x}_{1}\right) / \partial \bar{x}_{1}\right)\left(\phi_{1}\left(\bar{\zeta}_{1}, \bar{\chi}_{1}, d\right)+\alpha_{1}\left(\bar{x}_{1}\right)+\bar{x}_{2}\right) \\
& +\ell_{2}\left(y_{2}, s_{2}, \hat{\omega}_{1}, \mu\right)+u \\
= & \phi_{2}\left(\bar{\zeta}_{2}, \bar{\chi}_{2}, d\right)+\alpha_{2}\left(\bar{x}_{2}\right)-\rho_{2}\left(y_{2}, s_{2}, \hat{\omega}_{1}\right) \tilde{\omega}_{2}
\end{aligned}
$$


with $\tilde{\omega}_{2}=\hat{\omega}_{2}-\omega_{2}$. From the above development, the system 2.8 is put in the following form:

$$
\begin{aligned}
\dot{x}_{o} & =f_{o}\left(x_{o}, x_{1}, d\right) \\
\dot{\bar{z}}_{i} & =M_{i} \bar{z}_{i}+\varphi_{i}\left(\bar{\zeta}_{i-1}, \bar{\chi}_{i}, d\right) \\
\dot{\bar{x}}_{i} & =\phi_{i}\left(\bar{\zeta}_{i}, \bar{\chi}_{i}, d\right)+\alpha_{i}\left(\bar{x}_{i}\right)-\rho_{i}\left(y_{i}, s_{i}, \hat{\omega}_{i-1}\right) \tilde{\omega}_{i}+\bar{x}_{i+1}, \quad i=1,2
\end{aligned}
$$

under the following controller:

$$
\begin{aligned}
u & =-\rho_{2}\left(y_{2}, s_{2}, \hat{\omega}_{1}\right) \hat{\omega}_{2}+\alpha_{2}\left(\bar{x}_{2}\right) \\
\bar{x}_{2} & =x_{2}+\rho_{1}\left(y_{1}\right) \hat{\omega}_{1}-\alpha_{1}\left(x_{1}\right) \\
\dot{\hat{\omega}}_{1} & =\psi_{1}\left(y_{1}\right), \quad \dot{\hat{\omega}}_{2}=\psi_{2}\left(y_{2}, s_{2}, \hat{\omega}_{1}\right) \\
\dot{s}_{2} & =M_{2} s_{2}+\varrho_{2}\left(\bar{y}_{2}, \hat{\omega}_{1}\right)
\end{aligned}
$$

where $\hat{\omega}_{o} \in \mathbb{R}^{0}$. Now, it is ready to have the main result.

Theorem 3.1. Under Assumptions 2.1 to 2.4. form any initial condition, the states of the system (3.9) are bounded and $\lim _{t \rightarrow \infty} x_{1}(t)=0$. Therefore, the global robust adaptive output regulation problem for the system (1.1) is solved by the controller (3.10) and (2.3).

Proof. Compare the system (3.9) with the system (3.1), we can define a function

$$
U\left(\bar{\zeta}_{2}, \bar{\chi}_{2}, \tilde{\omega}\right)=V\left(\bar{\zeta}_{2}\right)+W\left(\bar{\chi}_{2}\right)+\frac{1}{2} \tilde{\omega}^{\top} K \tilde{\omega}
$$

where $\tilde{\omega}:=\operatorname{col}\left(\tilde{\omega}_{1}, \tilde{\omega}_{2}\right)$, and the matrix $K=\operatorname{diag}\left(k_{1}, k_{2}\right)$ is diagonal and positive definite which is used to modify the update rate. Using (3.2), it can be shown that the derivative of $U\left(\bar{\zeta}_{2}, \bar{\chi}_{2}, \tilde{\omega}\right)$ along the system 3.9$)$ is

$$
\frac{d U\left(\bar{\zeta}_{2}, \bar{\chi}_{2}, \tilde{\omega}\right)}{d t} \leq-k\left(\bar{\zeta}_{2}, \bar{\chi}_{2}\right)-\frac{\partial W\left(\bar{\chi}_{2}\right)}{\partial\left[\bar{x}_{1}, \bar{x}_{2}\right]^{\top}} \rho\left(y_{2}, s_{2}, \hat{\omega}_{1}\right) \tilde{\omega}+\tilde{\omega}^{\top} K \psi\left(y_{2}, s_{2}, \hat{\omega}_{1}\right)
$$

where

$$
\begin{aligned}
& \rho\left(y_{2}, s_{2}, \hat{\omega}_{1}\right)=\operatorname{block} \operatorname{diag}\left(\rho_{1}\left(y_{1}\right), \rho_{2}\left(y_{2}, s_{2}, \hat{\omega}_{1}\right)\right) \\
& \psi\left(y_{2}, s_{2}, \hat{\omega}_{1}\right)=\operatorname{col}\left(\psi_{1}\left(y_{1}\right), \psi_{2}\left(y_{2}, s_{2}, \hat{\omega}_{1}\right)\right) .
\end{aligned}
$$

Now, let the update law be

$$
\dot{\tilde{\omega}}=\psi\left(y_{2}, s_{2}, \hat{\omega}_{1}\right)=K^{-1}\left[\frac{\partial W\left(\bar{\chi}_{2}\right)}{\partial\left[\bar{x}_{1}, \bar{x}_{2}\right]^{\top}} \rho\left(y_{2}, s_{2}, \hat{\omega}_{1}\right)\right]^{\top} .
$$


As a result,

$$
\frac{d U\left(\bar{\zeta}_{2}, \bar{\chi}_{2}, \tilde{\omega}\right)}{d t} \leq-k\left(\bar{\zeta}_{2}, \bar{\chi}_{2}\right)
$$

Thus, the states $\bar{\zeta}_{2}, \bar{\chi}_{2}$, and $\tilde{\omega}$ are bounded. It is easy to verify that $s_{2}$ is bounded. In other words, all states in the closed-loop system are bounded. Next, let $U(t)=U\left(\bar{\zeta}_{2}(t), \bar{\chi}_{2}(t), \tilde{\omega}(t)\right)$ and $k(t)=k\left(\bar{\zeta}_{2}(t), \bar{\chi}_{2}(t)\right)$. Integrating on both sides of 3.12 shows that $\int_{o}^{t} k(\tau) d \tau \leq U(0)-U(t) \leq U(0)$, hence, $\lim _{t \rightarrow \infty} \int_{o}^{t} k(\tau) d \tau$ exists and is finite. The fact that all states are bounded implies that

$$
\dot{k}(t)=\frac{\partial k\left(\bar{\zeta}_{2}, \bar{\chi}_{2}\right)}{\partial \bar{\zeta}_{2}} \dot{\bar{\zeta}}_{2}+\frac{\partial k\left(\bar{\zeta}_{2}, \bar{\chi}_{2}\right)}{\partial \bar{\chi}_{2}} \dot{\bar{\chi}}_{2}
$$

is bounded for all $t \geq 0$, hence, $k(t)$ is uniformly continuous. By Barbalat's lemma, $\lim _{t \rightarrow \infty} k(t)=0$, hence $\lim _{t \rightarrow \infty} x_{1}(t)=0$. The proof is thus completed.

Remark 3.1. The stabilization problem of some special cases of the system 2.8 has been extensively studied over the past two decades. The simplest case where the system involves neither dynamic uncertainty nor static uncertainty was solved via full state feedback control using the well known backstepping technique in a series of papers [14-16]. When the system contains only the static uncertainty, the problem was also dealt with via state feedback control using the adaptive backstepping approach under various assumptions on the uncertainty vector $d(t)$ itself and the way $d(t)$ enters the system in [17 19], to name just a few. For the more general case where the system contains both static and dynamic uncertainties, a similar problem was also studied in several papers [13, 20, 21] by under the assumption that the dynamic uncertainty satisfies a certain input-to-state stability condition.

Remark 3.2. Some features of the proposed controller design approach are highlighted as follows. (i) As $z_{2}$ is not available for feedback, the standard adaptive control technique, e.g., [18], is incapable of handling the e.p.p. term $h_{2}$ appearing in the subsystem governing $z_{2}$. We have devised a dynamic coordinate transformation (3.6) to overcome this difficulty. (ii) At the second step, the uncertainties from the first step enter the e.p.p. terms $h_{2}$ and $l_{2}$ and hence induce more complex e.p.p. terms $\hbar_{2}$ and $\ell_{2}$. This phenomenon is called propagation of uncertainty. As a result, we need to examine the linearly parameterization property of these new terms. This examination may become more difficult if a system with a higher relative degree is considered. 
Remark 3.3. From (3.11), it is known that the rate of the parameter estimate converges to zero since $\lim _{t \rightarrow \infty} \bar{\chi}_{2}(t)=0$. Moreover, we can give the condition under which the parameter $\hat{\omega}_{i}$ converges to the real value $\omega_{i}(\mu)$, i.e., $\lim _{t \rightarrow \infty} \tilde{\omega}_{i}(t)=0$. To give that condition, we note that $\lim _{t \rightarrow \infty} \bar{x}_{i}(t)=$ 0 and $\dot{d}(t)$ is bounded, then $\ddot{\bar{x}}_{i}(t)$ is bounded, that is, $\dot{\bar{x}}_{i}(t)$ is uniformly continuous in $t$. By Barbalat's lemma, $\lim _{t \rightarrow \infty} \dot{\bar{x}}_{i}(t)=0$. As a result, we have $\lim _{t \rightarrow \infty} \rho_{i}(t) \tilde{\omega}_{i}(t)=0$. Using Lemma 4.1 of [11], we have $\lim _{t \rightarrow \infty} \tilde{\omega}_{i}(t)=$ 0 if $\rho_{i}^{\top}(t)$ is a persistent excitation (PE). For the system (1.1), we note that $\rho_{1}^{\top}(t)=-\bar{\Psi}_{1} \eta_{1}(t)$ is a PE if $\eta_{1}(t)$ is. Thus, the estimated parameter $\hat{\omega}_{1}$ converges to its true value $\omega_{1}(\mu)$ if $\theta_{1}(t)$ is a $\mathrm{PE}$.

Example 3.1. We consider the global output regulation problem of the following system:

$$
\begin{aligned}
& \dot{q}_{o}=-q_{o}+0.2 e, \quad \dot{q}_{1}=0.5 q_{o} \sin q_{1}+w_{1} q_{1}+q_{2} \\
& \dot{q}_{2}=w_{2} q_{o}^{2}+w_{3} q_{1}+u, \quad e=q_{1}-v_{1}
\end{aligned}
$$

coupled with an exosystem

$$
\dot{v}_{1}=-\sigma v_{2}, \quad \dot{v}_{2}=\sigma v_{1}
$$

These equations formulate the control problem of designing a state feedback regulator to have the output $x_{1}$ of system (3.13) asymptotically track a sinusoidal signal of an unknown frequency with an arbitrarily large fixed amplitude, produced by the exosystem (3.14), in the presence of three uncertain parameters $\left(w_{1}, w_{2}, w_{3}\right)$. In this example, we allow a non-polynomial term $\sin q_{1}$ in the system to show that the polynomial assumption is not necessary as long as there exists a polynomial solution to the regulator equations.

It can be verified that Assumptions 2.1 2.4 are satisfied. In particular, the regulator equations associated with (3.13) and (3.14) have a globally defined solution in polynomial form as follows

$$
\begin{aligned}
& \mathbf{q}_{o}(v, w, \sigma)=0, \quad \mathbf{q}_{1}(v, w, \sigma)=v_{1} \\
& \mathbf{q}_{2}(v, w, \sigma)=-\sigma v_{2}-w_{1} v_{1}, \quad \mathbf{u}(v, w, \sigma)=-\sigma^{2} v_{1}+w_{1} \sigma v_{2}-w_{3} v_{1} .
\end{aligned}
$$

To construct the internal model, we have the matrices

$$
\begin{aligned}
\Phi_{1}(\sigma) & =\Phi_{2}(\sigma)=\left[\begin{array}{cc}
0 & 1 \\
-\sigma^{2} & 0
\end{array}\right] \\
M_{1} & =M_{2}=\left[\begin{array}{cc}
-1 & 0 \\
0 & -2
\end{array}\right], \quad N_{1}=N_{2}=\left[\begin{array}{l}
0.2 \\
0.5
\end{array}\right] .
\end{aligned}
$$


Next, we can solve $T_{i}(\sigma)$ and $\Psi_{i}(\sigma)$ as

$$
\begin{aligned}
& T_{1}(\sigma)=T_{2}(\sigma)=\left[\begin{array}{ll}
\frac{0.2}{\sigma^{2}+1} & -\frac{0.2}{\sigma^{2}+1} \\
\frac{1}{\sigma^{2}+4} & -\frac{0.5}{\sigma^{2}+4}
\end{array}\right], \\
& \Psi_{1}(\sigma)=\Psi_{2}(\sigma)=\left[\begin{array}{ll}
-5 \sigma^{2}-5 & 2 \sigma^{2}+8
\end{array}\right] .
\end{aligned}
$$

After the introduction of the internal model 2.3 , we can define an augmented system and further transform the augmented system into the form (2.8). Next, we will give the detailed calculation on the quantities used in the controller design. First, we can choose

$$
\alpha_{1}\left(x_{1}\right)=-K_{1} x_{1}, \quad \alpha_{2}\left(\tilde{x}_{2}\right)=-K_{2} \tilde{x}_{2}\left(1+\tilde{x}_{2}^{2}\right), \quad p_{1}=2, \quad p_{2}=1
$$

where $K_{1}$ and $K_{2}$ are determined by the bound of $w_{1}, w_{2}, w_{3}$ and $\sigma$. Since $l_{1}\left(y_{1}, \mu\right)=-\Psi_{1}(\sigma) \eta_{1}$, we can verify that

$$
\rho_{1}\left(y_{1}\right)=-\eta_{1}^{\top} \bar{\Psi}_{1}^{\top}, \quad \bar{\Psi}_{1}=\left[\begin{array}{ll}
-5 & 2 \\
-5 & 8
\end{array}\right], \quad \omega_{1}(\mu)=\operatorname{col}\left(\sigma^{2}, 1\right) .
$$

Next, note $\Psi_{1}(\sigma) E_{1}(\sigma)=\left[-10 \sigma^{2}-10,2 \sigma^{2}+8\right]$. Since

$$
\hbar_{2}\left(\bar{y}_{2}, \hat{\omega}_{1}, \mu\right)=M_{2} N_{2} \eta_{1}^{\top} \bar{\Psi}_{1}^{\top} \hat{\omega}_{1}-N_{2} \Psi_{1}(\sigma) E_{1}(\sigma) \eta_{1}
$$

we can verify that

$$
\begin{aligned}
\varrho_{2}\left(\bar{y}_{2}, \hat{\omega}_{1}\right) & =\left[\begin{array}{ll}
0 & M_{2} N_{2} \eta_{1}^{\top} \bar{\Psi}_{1}^{\top} \hat{\omega}_{1}
\end{array}\right]-N_{2} \eta_{1}^{\top} \hat{\Psi}_{1}^{\top}, \\
\hat{\Psi}_{1} & =\left[\begin{array}{ll}
-10 & 2 \\
-10 & 8
\end{array}\right], \quad \varpi_{2}(\mu)=\operatorname{col}\left(\sigma^{2}, 1\right) .
\end{aligned}
$$

We note the derivative of $\rho_{1}\left(y_{1}\right)$ is $\bar{\rho}_{1}\left(y_{2}, \mu\right)=-\dot{\eta}_{1}^{\top} \bar{\Psi}_{1}^{\top}=-\left(M_{1} \eta_{1}+N_{1} x_{2}\right)^{\top} \bar{\Psi}_{1}^{\top}$, which is well defined and measurable. A calculation shows

$$
\ell_{2}\left(y_{2}, \xi_{2}, \hat{\omega}_{1}, \mu\right)=\left[A_{11} A_{12}+A_{21} A_{22}\right] \operatorname{col}\left(\sigma^{4}, \sigma^{2}, 1\right)+B \operatorname{col}\left(\sigma^{2}, 1\right)+C
$$

with

$$
\begin{aligned}
& A=\bar{\Psi}_{1} s_{2} \\
& B=-\left(N_{2} \rho_{1}\left(y_{1}\right) \hat{\omega}_{1}\right)^{\top} \bar{\Psi}_{1}^{\top}+\eta_{1}^{\top} \hat{\Psi}_{1}^{\top}-\eta_{2}^{\top} \bar{\Psi}_{1}^{\top}-\left(\partial \alpha_{1}\left(\bar{x}_{1}\right) / \partial \bar{x}_{1}\right) \rho_{1}\left(y_{1}\right) \\
& C=\left(\partial \alpha_{1}\left(\bar{x}_{1}\right) / \partial \bar{x}_{1}\right) \rho_{1}\left(y_{1}\right) \hat{\omega}_{1}+\bar{\rho}_{1}\left(y_{2}, \mu\right) \hat{\omega}_{1}+\rho_{1}\left(y_{1}\right) \psi_{1}\left(y_{1}\right) .
\end{aligned}
$$


Therefore,

$$
\begin{aligned}
\rho_{2}\left(y_{2}, s_{2}, \hat{\omega}_{1}\right) & =\left[\begin{array}{ll}
0 & B
\end{array}\right]+\left[\begin{array}{lll}
A_{11} & A_{12}+A_{21} & C+A_{22}
\end{array}\right], \\
\omega_{2} & =\operatorname{col}\left(\sigma^{4}, \sigma^{2}, 1\right) .
\end{aligned}
$$

In the numerical simulation, we can compare the non-adaptive controller and the adaptive one. The simulation is conducted with the parameters $w_{1}=-0.4, w_{2}=0.8, w_{3}=0.3, v_{1}(0)=10, v_{2}(0)=0, q_{o}(0)=5, q_{1}(0)=$ $8, q_{2}(0)=-1$, and the initial values of the remaining states being zero. The simulation condition is listed in Table1. For the first 100 seconds, the value

\begin{tabular}{|c||c|c|c|c|}
\hline time(s) & $0-100$ & $100-200$ & $200-300$ & $300-400$ \\
\hline adaptive law & off & off & on & on \\
\hline$\sigma$ & 0.2 & 1 & 1 & 0.2 \\
\hline
\end{tabular}

Table 1: Simulation condition for the system (3.13).

of $\sigma$ is the same as that used for the controller design, the adaptive law is off. The desired tracking performance $\lim _{t \rightarrow \infty} e(t)=0$ is shown in Figure 1 . At $t=100$, the parameter $\sigma$ changes its value and the tracking performance degrades significantly. When the adaptive law is turned on at $t=200$, the tracking error quickly converges to zero. Good tracking performance is maintained even after another step change of the parameter at $t=300$. The tracking performance is shown in Figure. 1 in a similar tendency.

Also, we can check that the signal

$$
\theta_{1}(v, w, \sigma)=T_{1}(\sigma)\left[\begin{array}{cc}
-w_{1} & -\sigma \\
-\sigma^{2} & \sigma w_{1}
\end{array}\right]\left[\begin{array}{l}
v_{1} \\
v_{2}
\end{array}\right]
$$

is a $\mathrm{PE}$ if $v(t) \neq 0$. Then, the parameter estimation $\hat{\omega}_{1}$ always converges to its real value $\omega_{1}(\mu)$. Since the unknown frequency $\sigma$ is related to $\hat{\omega}_{11}, \hat{\omega}_{21}$, and $\hat{\omega}_{22}$ by $\hat{\sigma}_{1}=\sqrt{\hat{\omega}_{11}}, \hat{\sigma}_{2}=\sqrt[4]{\hat{\omega}_{21}}$, and $\hat{\sigma}_{3}=\sqrt{\hat{\omega}_{22}}$, any one of the three relations gives the precise estimation of $\sigma$ as shown in Figure 2 .

\section{Conclusion}

A common feature of the existing approaches to handling the adaptive output regulation problem with uncertain exosystems is that the internal model compensator only consists of a single dynamic compensator corresponding to the input. In contrast, in this paper, we have to construct an internal model 

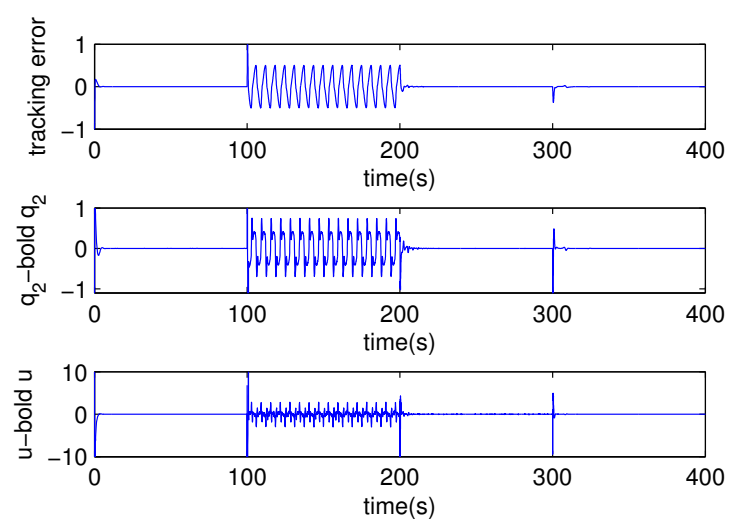

Figure 1: Profile of the tracking errors for the plant states and input.
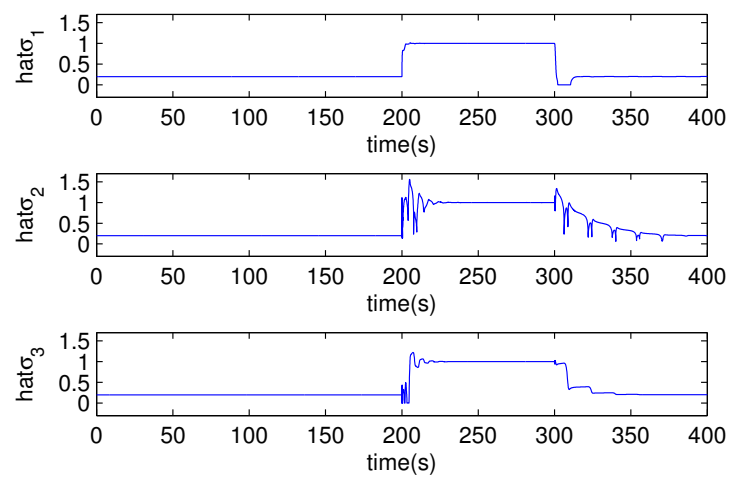

Figure 2: Profile of the estimated frequencies.

consisting of two dynamic compensators corresponding to the input and the state $q_{2}$, respectively. As a result, we have to deal with a much more complicated augmented system. We have proposed a recursive design method to solve the problem. Even though we have only considered a lower triangular system with relative degree two, the relative degree one case can be considered as a special case. Moreover, the recursive nature of our approach allows it to be extended to systems with a higher relative degree at the cost of more complicated notation and assumptions. 


\section{Acknowledgements}

This work was supported by the Research Grants Council of the Hong Kong Special Administration Region under grant No. 412813.

\section{References}

[1] A. Isidori and C. I. Byrnes, Output regulation of nonlinear systems. IEEE Transactions on Automatic Control, 35 (1990), 131-140.

[2] H. Khalil, Robust servomechanism output feedback controllers for feedback linearizable systems. Automatica, 30 (1994), 1587-1589.

[3] C. I. Byrnes and A. Isidori, Limit sets, zero dynamics and internal models in the problem of nonlinear output regulation. IEEE Transactions on Automatic Control, 48 (2003), 1712-1723.

[4] Z. Ding, Universal disturbance rejection for nonlinear systems in output feedback form. IEEE Transactions on Automatic Control, 48 (2003), 1222-1226.

[5] J. Huang and Z. Chen, A general framework for tackling the output regulation problem. IEEE Transactions on Automatic Control, 49 (2004), 2203-2218.

[6] V. O. Nikiforov, Adaptive non-linear tracking with complete compensation of unknown disturbances. European Journal of Control, 4 (1998), $132-139$.

[7] X. D. Ye and J. Huang, Decentralized adaptive output regulation for a class of large-scale nonlinear systems. IEEE Transactions on Automatic Control, 48 (2003), 276-281.

[8] Z. Ding, Global stabilization and disturbance suppression of a class of nonlinear systems with uncertain internal model. Automatica, 39 (2003), 471-479.

[9] Z. Ding, Adaptive estimation and rejection of unknown sinusoidal disturbances in a class of non-minimum-phase nonlinear systems. IEE Proc.-Control Theory Appl., 153 (2006), 379-386.

[10] R. Marino and P. Tomei, Adaptive nonlinear regulation for uncertain minimum phase systems with unknown exosystem. Proceedings of the 47th IEEE Conference on Decision and Control, pages 2569-2574, 2008. 
[11] L. Liu, Z. Chen and J. Huang, Parameter convergence and minimal internal model with an adaptive output regulation problem. Automatica, 45 (2009), 1306-1311.

[12] A. Serrani, A. Isidori and L. Marconi, Semiglobal nonlinear output regulation with adaptive internal model. IEEE Transactions on Automatic Control, 46 (2001), 1178-1194.

[13] Z. Chen and J. Huang, A Lyapunov's direct method for the global robust stabilization of nonlinear cascaded systems. Automatica, 44 (2008), 745-752.

[14] C. Byrnes and A. Isidori, New results and examples in nonlinear feedback stabilization. Systems and Control Letters, 12 (1989), 437-442.

[15] I. Kanellakopoulos, P. Kokotović and A. S. Morse, A toolkit for nonlinear feedback design. Systems and Control Letters, 18 (1992), 83-92.

[16] J. Tsinias, Sufficient Lyapunov-like conditions for stabilization. Math. Contr. sign. Syst., 2 (1989), 343-357.

[17] Z. P. Jiang, A. R. Teel and L. Praly, Small-gain theorem for ISS systems and applications. Mathematics of Control, Signals and Systems, 7 (1994), 95-120.

[18] Z. P. Jiang and L. Praly, Design of robust adaptive controllers for nonlinear systems with dynamic uncertainties. Automatica, 34 (1998), 825-840.

[19] H. Ito, A constructive proof of ISS small-gain theorem using generalized scaling. Proceedings of the 41st IEEE Conference on Decision and Control, pages 2286-2291, Dec. 2002.

[20] Z. P. Jiang and I. Mareels, A small-gain control method for nonlinear cascaded systems with dynamic uncertainties. IEEE Transactions on Automatic Control, 42 (1997), 292-308.

[21] Z. Chen and J. Huang, Dissipativity, stabilization, and regulation of cascade-connected systems. IEEE Transactions on Automatic Control, 49 (2004), 635-650. 
School of Electrical Engineering and Computer Science The University of NewCAstle

Callaghan, NSW 2308, Australia

E-mail address: zhiyong.chen@newcastle.edu.au

Department of Mechanical and Automation Engineering The Chinese University of Hong Kong

Shatin, N.T., Hong Kong, China

E-mail address: jhuang@mae.cuhk.edu.hk

RECEIVED MAY 21, 2012

Accepted October 12, 2015 
